\title{
Mode-Locked Topological Insulator Laser Utilizing Synthetic Dimensions
}

\author{
Zhaoju Yang, ${ }^{\dagger}$ Eran Lustig, ${ }^{\dagger}$ Gal Harari, Yonatan Plotnik, Yaakov Lumer, Miguel A. Bandres®, and Mordechai Segev ${ }^{*}$ \\ Physics Department and Solid State Institute, Technion-Israel Institute of Technology, Haifa 32000, Israel
}

(Received 28 May 2019; revised manuscript received 2 December 2019; accepted 20 December 2019; published 9 March 2020)

\begin{abstract}
We propose a system that exploits the fundamental features of topological photonics and synthetic dimensions to force many semiconductor laser resonators to synchronize, mutually lock, and under suitable modulation emit a train of transform-limited mode-locked pulses. These lasers exploit the Floquet topological edge states in a 1D array of ring resonators, which corresponds to a $2 \mathrm{D}$ topological system with one spatial dimension and one synthetic frequency dimension. We show that the lasing state of the multielement laser system possesses the distinct characteristics of spatial topological edge states while exhibiting topologically protected transport. The topological synthetic-space edge mode imposes a constant-phase difference between the multifrequency modes on the edges, and together with modulation of the individual elements forces the ensemble of resonators to mode lock and emit short pulses, robust to disorder in the multiresonator system. Our results offer a proof-of-concept mechanism to actively mode lock a laser diode array of many lasing elements, which is otherwise extremely difficult due to the presence of many spatial modes of the array. The topological synthetic-space concepts proposed here offer an avenue to overcome this major technological challenge and open new opportunities in laser physics.
\end{abstract}

DOI: 10.1103/PhysRevX.10.011059

Subject Areas: Optics, Photonics, Topological Insulators

Topological insulators [1,2] are a new phase of matter with an insulating bulk that supports topologically protected unidirectional edge states. These edge states are endowed with topological invariants and their transport is therefore robust against defects and disorder as long as the topological band gap is preserved. Since their observation in electronic systems [3], topological insulators have been studied in many physical systems such as microwaves [4-6], photonics [7-14], acoustics [15,16], and mechanics $[17,18]$. Outside condensed matter, topological insulators were first proposed to occur also with electromagnetic (EM) waves $[4,5]$, and shortly thereafter observed with microwaves in gyro-optic materials [6]. At that point it was clear that introducing the topological concepts into photonics would lead to many new discoveries. However, this transition turned out to be difficult because at optical frequencies the gyro-optic response to magnetic fields is extremely weak, resulting in only a tiny topological band gap that would be robust only against extremely weak perturbations. Thus, the realization of photonic topological insulators had to rely on artificial magnetic fields [19]

\footnotetext{
*Corresponding author. msegev@technion.ac.il

These authors contributed equally to this work.

Published by the American Physical Society under the terms of the Creative Commons Attribution 4.0 International license. Further distribution of this work must maintain attribution to the author(s) and the published article's title, journal citation, and DOI.
}

rather than on magneto-optics. In the following years, several ideas were proposed along these lines $[8,10,13,20,21]$, until eventually photonic topological insulators were demonstrated experimentally in 2013 $[11,12]$. Both of these systems relied on photonic crystal settings incorporating artificial gauge fields. However, topological insulators do not necessarily have to rely on spatial dimensions. Rather, topological phenomena can be constructed in synthetic dimensions [22-24], using internal degrees of freedom such as spin in atomic systems [25,26], or by using external degrees of freedom such as shaking harmonic traps containing cold atoms [27]. In photonics, synthetic dimensions were proposed for generating solitons in higher dimensions [28], and were demonstrated in the context of topological pumping $[29,30]$. In this vein, topological insulators were proposed to occur in photonic platforms utilizing synthetic space, such as the modal dimension of a resonator [31-33], with intriguing suggestions for observing high-dimensional quantum Hall effect and Weyl points [31,34] by controlling the phase of the dynamic modulation [9]. Eventually, in 2019, photonic topological insulators in synthetic dimensions were demonstrated experimentally, by utilizing a lattice of spatially modulated waveguides [35]. Soon thereafter, synthetic Hall ladders were demonstrated in a ring resonator, also manifesting topological transport in synthetic dimensions [36].

The unique features of topological photonics have led to many new applications where robustness is important, such as delay lines [12], or recently, topological insulator lasers [37-39], which includes gain within the laser cavity and 
utilizes the topological edge states as the lasing mode. Topological insulator lasers combine the concepts of topological insulators with the fundamentals of lasers, which introduce gain, loss, and demonstrate lasing action in a topological platform. Specifically, in the presence of an artificial gauge field [38], an optical lattice consisting of resonators exhibits topological edge states. Incorporating gain on the edges of the 2D lattice of coupled resonators causes the topological edge mode to lase, displaying unidirectional energy flux, high slope efficiency, and single-mode lasing even high above the laser threshold [37,38]. More recently, these concepts of topological insulator laser have led to topological crystalline bulk lasers [40] and to electrically pumped topological Valley-Hall quantum cascade laser systems [41]. These concepts offer many new research directions, ranging from new designs for masers [42], phonon lasers [43], spacers [44-46], and lasing action in the recently discovered exciton-polariton topological insulators $[47,48]$.

Here, we take the concept of topological insulator laser to the next level and propose multielement mode-locked topological insulator lasers in synthetic dimensions.

Let us first explain the technological and conceptual challenge to mode lock a laser diode array. An array of evanescently coupled laser diodes was proposed 35 years ago as an avenue to achieve a coherent high-power laser source based on semiconductor technology [49,50]. However, within a decade it became clear that semiconductor laser arrays tend to lase in many modes simultaneously, that their field pattern and spectra vary considerably even under small variations in the gain, and that the mutual coherence of the array is greatly reduced with increasing the number of laser elements. Consequently, current technology of laser arrays is limited to acting as a pump for solid-state lasers [51], but not as a high-power coherent laser source, as was originally envisioned. Another major consequence of the extreme difficulty in locking multiple elements of semiconductor lasers is the inability to synchronize them, that is, to generate mode-locked pulses from a laser diode array. The difficulty arises from the fact that a laser diode array has multiple spatial modes (array modes), and each spatial mode has a set of associated resonance frequencies that are shifted from those of the other array modes. In addition, the spectra of modulated laser diodes is fundamentally chirped (i.e., the spectrum varies during the modulation) [52,53] and the chirp of different laser elements is sensitive to small fabrication differences. On the other hand, achieving mode locking requires synchronizing all the lasing frequencies of all the lasing elements, which becomes extremely hard when the laser has multiple spatial modes, as all laser arrays fundamentally have. For this reason, the only attempts to mode lock a laser diode array relied on having external cavities [54-56], and even those were left unfollowed, never maturing into real technology.
In contrast to the early attempts to actively mode lock laser diode arrays, we propose a system that exploits the fundamental features of topological photonics to force many semiconductor laser resonators to synchronize, mutually lock, and emit a train of transform-limited mode-locked pulses. Our proposed system relies on a topological platform that utilizes a Floquet topological edge state in a 2D synthetic space that is composed of one modal dimension (the modes of the resonators) and one spatial dimension (the array of resonators). The edge states live on a closed path encircling a $2 \mathrm{D}$ region in synthetic space. The synthetic dimension here is a ladder of equally spaced modes (frequencies). Thus, unlike a real-space topological insulator laser (which can lase at a single frequency), here the spectrum of a topological edge state is a frequency comb. This setting, together with gain saturation (present in all lasers), selects a single lasing mode - the Floquet topological edge mode-for the entire array, which has a single set of equally spaced resonance frequencies. This unidirectionally propagating lasing state in synthetic dimensions is robust to imperfections and small variations in the individual laser elements. It induces a fixed phase difference between the modes of the cavity and therefore generates constructive interference between these modes, causing the laser light to be produced as transform-limited mode-locked pulses.

To construct the topological laser in synthetic dimensions, we adopt the modulated ring resonators $[31,33,57]$ as our building block. In the absence of group velocity dispersion in the underlying ambient medium, each ring resonator supports a discrete set of resonant modes at equally spaced frequencies described by $\omega_{m}=\omega_{0}+m \Omega$, where $\Omega$ is the free spectral range of the ring, $\omega_{0}$ is the offset frequency, and $m$ is an integer. Each ring is coupled to two neighboring rings, and we assume static coupling only between modes with the same frequency at the nearest neighbor resonators, with a coupling strength $t_{0}$. In addition, each resonator is modulated with the frequency $\Omega$, which induces dynamic coupling (coupling strength $t_{f}$ ) between modes separated by $\Omega$. Combined with the mode dimension, the tight-binding Hamiltonian [33] of the system can be expressed as

$$
\begin{aligned}
H= & \sum_{j, m} \omega_{m} a_{j, m}^{\dagger} a_{j, m}+\sum_{j, m} t_{0}\left(a_{j, m}^{\dagger} a_{j+1, m}+a_{j+1, m}^{\dagger} a_{j, m}\right) \\
& +\sum_{j, m} 2 t_{f} \cos \left(\Omega t+\phi_{j}\right)\left(a_{j, m}^{\dagger} a_{j, m+1}+a_{j, m+1}^{\dagger} a_{j, m}\right),
\end{aligned}
$$

where $a_{j, m}\left(a_{j, m}^{\dagger}\right)$ is the annihilation (creation) operator at the $j$ th site for mode $m, t$ is time, and $\phi_{j}$ is the modulation phase at the $j$ th resonator. If we set the modulation phase to be $\phi_{j}=x \phi$ ( $x$ is the unitless position of the resonator where each two neighboring resonators are separated by a distance 1 ), under the condition of $t_{f} \ll \Omega$ and the rotatingwave approximation, the dynamics of this photonic system 
can be mapped to the Hofstadter model [58] in a space with one spatial dimension and one modal dimension. Considering a strip geometry (finite number of rings and infinite modes in frequency dimension), we prove the existence of a topological band structure and topological edge states, as shown in Sec. A in the Supplemental Material [59].

Our design for the mode-locked topological laser system in synthetic dimensions is made up of identical modules, connected in a way that maintains their topological synthetic-space features. We first define the single module and then describe the full laser array system. The single module consists of two rows of evanescently coupled resonators [Fig. 1(a)]. The rows are decoupled from one another, but they are connected through the two side resonators. In total there are $N$ resonators, each of which supports $M$ equally spaced modes which can interact with light within the bandwidth of their active medium. The two-dimensional shaded regions green for the upper row of resonators, grey for the lower row of resonators, and yellow for the side resonators describe the synthetic space, which includes the position and the mode number. In the absence of gain and loss, this system displays a topological phase when $\phi \neq 0, \pi$, whereas for $\phi=0, \pi$, the system is topologically trivial. The topological band gap of the maximum size corresponds to $\phi=\pi / 2$. In the topological phase, this system exhibits unidirectional edge states spanning across the band gaps. To facilitate lasing of the edge state, we introduce optical gain and loss in the synthetic space, in the positions marked by blue and gray circles, respectively. More specifically, we introduce gain in mode 1 in the lower row and in mode $M$ in the upper row. The side ring has gain on all modes (the gain is homogenous on the side rings). In our structure, the modes with gain form a closed loop in synthetic space. If we assume, for simplicity, a cut-off [60] in the coupling above mode $M$, this closed loop overlaps with the edge-states of the system. In Sec. E in Supplemental Material [59], we show that this cut-off is not a necessary condition for the operation of our topological laser array system. The evolution of the field in this laser system is governed [37] by

$$
i \partial_{t} \boldsymbol{\Psi}=H \boldsymbol{\Psi}+\left(\frac{i g \mathbb{P}}{1+\frac{\sum_{m}\left|\varphi_{m}\right|^{2}}{I_{\mathrm{sat}}}}-i \gamma\right) \boldsymbol{\Psi},
$$

where $H$ indicates the Hamiltonian of the passive platform of the setup shown in Fig. 1(a) and $\boldsymbol{\Psi}$ is the column field vector for the amplitudes of all the relevant modes in the resonators $\left(\varphi_{m}\right.$ indicates the laser field in $m$ th synthetic mode). The second term includes the gain and linear loss $\gamma$ in each resonator, which manifests the fact that the lasers are fundamentally non-Hermitian and nonlinear entities. The optical gain via stimulated emission is inherently saturable $\left(I_{\text {sat }}\right)$, and the summation is taken over all modes of each resonator the gain in Eq. (2) is assumed to have homogenous broadening. However, all the calculations were carried out for both type of broadening, and yields similar results (in Sec. G in Supplemental Material [59] we show that inhomogeneous broadening performs slightly better). Here, $\mathbb{P}$ stands for the spatial profile of the pump. The gain $(g)$ is provided only to the modes on the perimeter of the synthetic space [blue circles in Fig. 1(a)]. Here the (a)

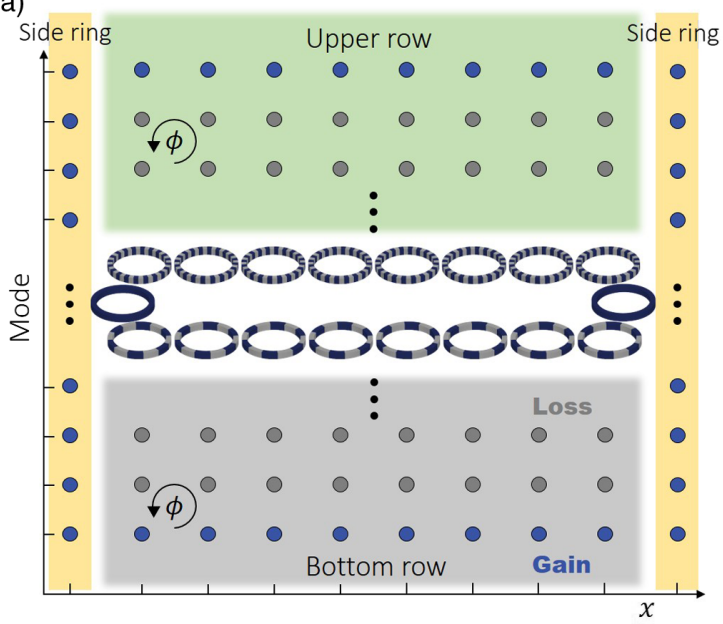

(b)

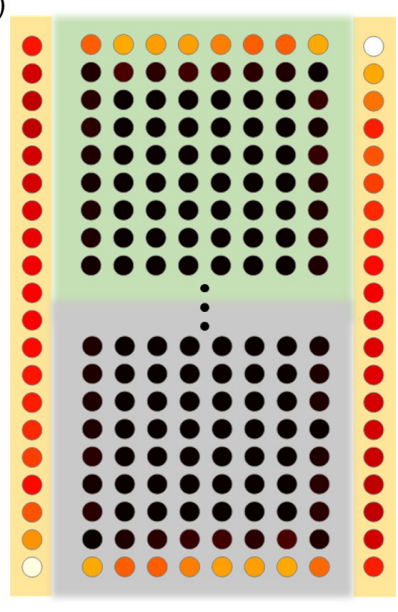

FIG. 1. Schematic of one module of the topological insulator laser in synthetic dimensions. (a) The photonic array consists of modulated rings, in the form of two independent rows connected by two side resonators. The blue and white staggered pattern indicates the different pumping schemes. The shaded regions show the synthetic space including one spatial and one synthetic mode dimensions. The blue (gray) circles indicate the gain (loss), where the gain is provided only to the upper (lower) mode in the upper (lower) row, and to all modes in the side rings. (b) The typical topological lasing state extending over the artificial perimeter of the $2 \mathrm{D}$ synthetic space. The color bar indicates the intensity of light. The unitless parameters for simulation are $N=18, M=20, t_{0}=t_{f}=1, \Omega=100, I_{\mathrm{sat}}=1$, and $\gamma=0.3$. The topological system has band gap size of $1.6 t_{0}$. 
gain profile is assumed to be broadband and does not depend on the modulation. Note that, since the gain saturation intensity for each resonator is the same and the edge state in synthetic dimensions occupies multiple modes of the side resonator, we need to accordingly pump the side resonators harder for smoothening the nonlinearityinduced potential barrier between the side resonator and its neighbors. We optimize and set the gain of side resonators $M / 2$ times larger than that of the bulk resonators through parameter sweeping.

The dynamics in this array of resonators is simulated by solving Eq. (2) numerically starting from random amplitudes and phases. As shown in Fig. 1(b), the topological lasing state $(\phi=\pi / 2)$ localizes at the edges of the synthetic space, which amounts to populating all the modes of the two side resonators, as well as the lowermost mode of the first row and the uppermost mode of the second row. Accordingly, we pump these modes in the pertinent resonators to obtain maximum overlap of the gain with the edge modes. In the real space picture, the light in the lasing state propagates through all the resonators and saturates the gain. In the synthetic space picture, this lasing state corresponds to the topological edge state on the perimeter of a $2 \mathrm{D}$ lattice with a perpendicular magnetic field. By contrast, in the corresponding topologically trivial system (same system with $\phi=0$ ), the lasing state stays almost stationary and penetrates deeper into the lossy bulk, which leads to lower lasing efficiency (see Sec. B in Supplemental Material [59]). Figure 2(a) displays the simulated laser output versus pumping intensity, with the blue (gray) describing the operation of the topologically nontrivial (trivial) laser. The slope efficiency of the topological laser is about twice that of the trivial laser.

Endowed with the topological properties, we expect the lasing state to exhibit robust transport associated with topological edge states. To examine this, we introduce fabrication disorder into the laser system, in the form of random variations in the resonant frequencies expressed as on-site disorder in the tight-binding model (with magnitude in units of the coupling coefficient). As shown in Fig. 2(b), (a)

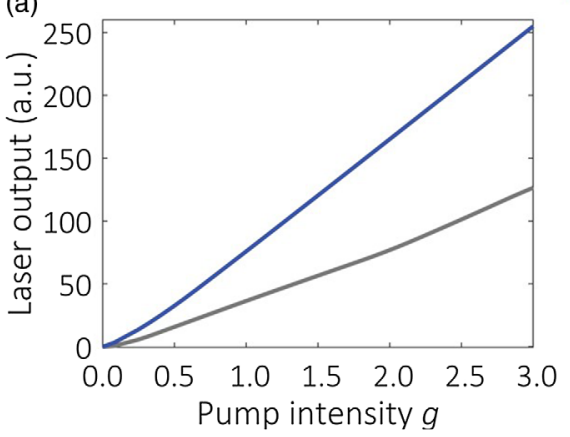

(b)

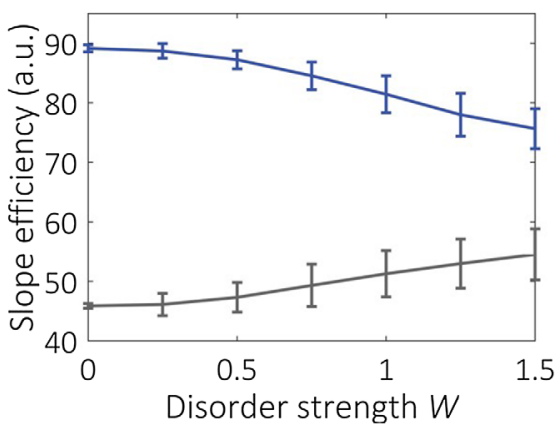

(c)
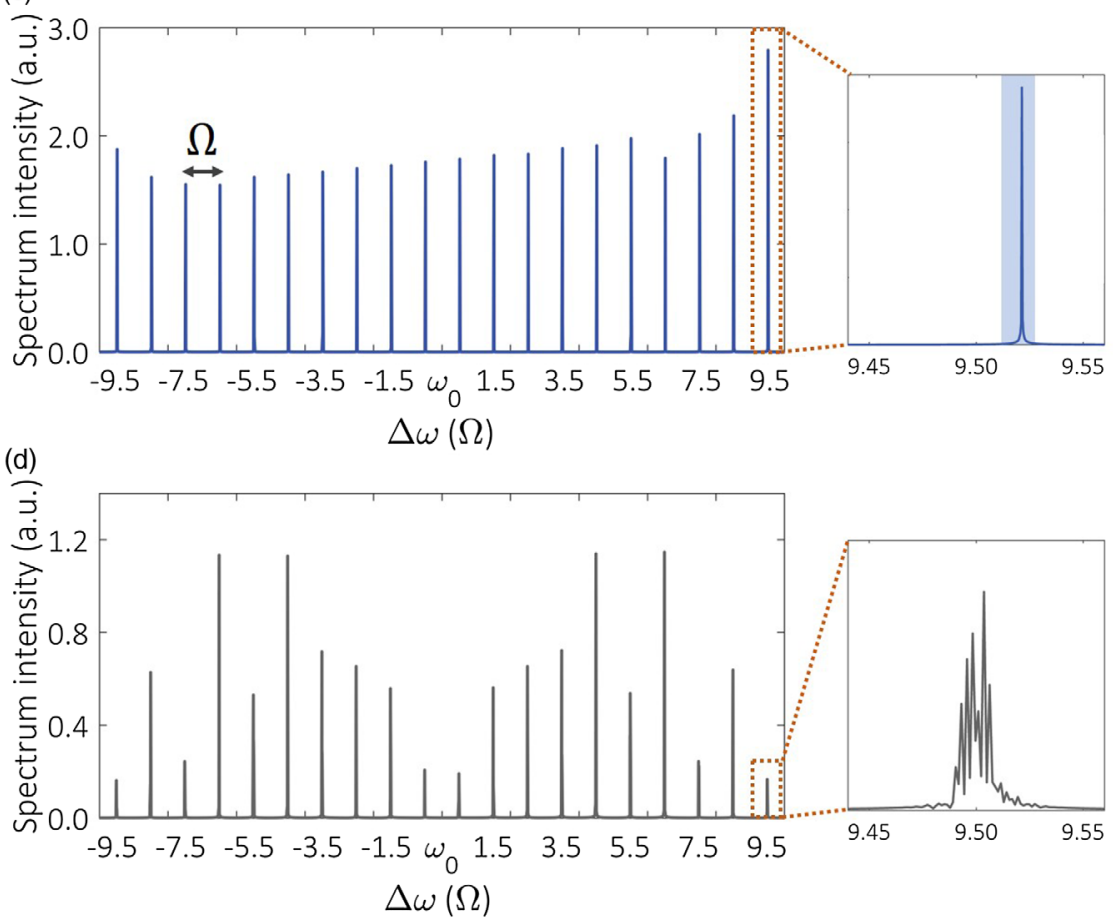

FIG. 2. Laser output versus (in arbitrary units, arb. units) pumping intensity and the spectra of the topologically nontrivial and trivial lasers. (a) The total output power versus pumping intensity. The slope efficiency of the topological laser is about twice that of the trivial laser. (b) Slope efficiency versus disorder strength. The on-site disorder is composed of independent random variations in the resonance frequencies, uniformly distributed in $[-W, W]$, where $W$ is in the units of $t_{0}$. This panel presents the mean of 100 realizations of disorder for every point of disorder strength. (c),(d) Frequency spectra of the topologically nontrivial (c) and trivial (d) lasers, as emitted from the connecting side resonator on the right-hand side; the panels on the right show the enlarged spectra around $9.5 \Omega$. The topological system emits a comb of narrow single frequencies, whereas the trivial system has multiple modes around each central frequency. The original resonances in each ring are set to be $-(M-1 / 2) \Omega, \ldots,(M-1 / 2) \Omega$ centered at $\omega_{0}(\Omega=100$ is the free spectral range, $M=20$ is the number of modes in each ring; $\omega_{0}$ is set to 0 for simplicity). The topological landscape causes a constant shift to all the resonance frequencies by a value of $0.019 \Omega$, as shown by the shift from $9.5 \Omega$ in the enlarged spectrum in (c) (see explanation in Sec. A in Supplemental Material [59]). The blue and gray curves represent the topological and trivial lasers, respectively. The blue shaded region on the right in (c) indicates the topological band gap. 

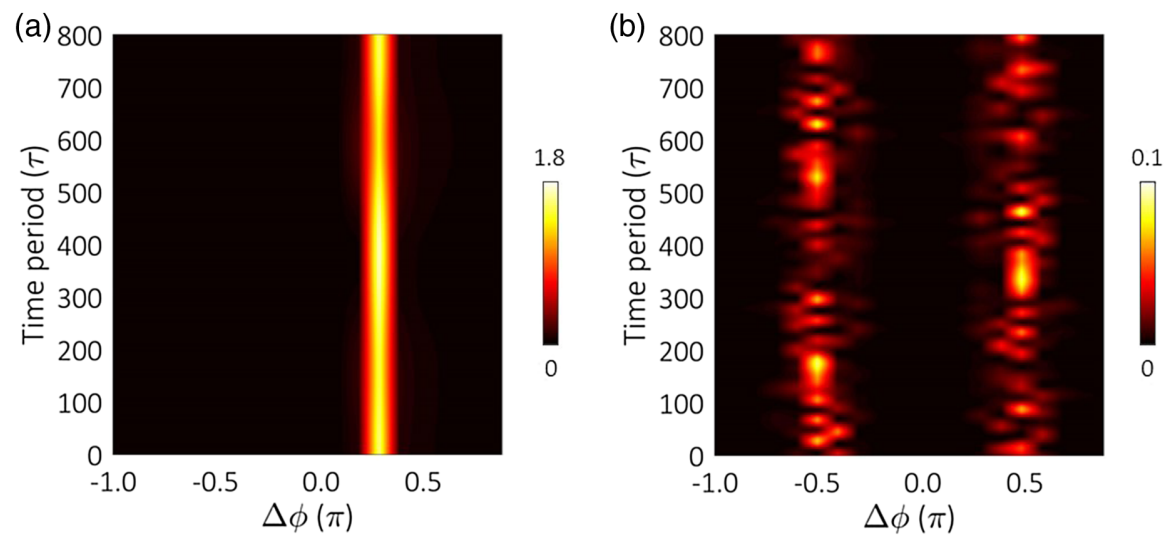

FIG. 3. Temporal dynamics of the phase difference between adjacent resonant modes in the side rings. The color bar indicates the absolute value squared of the spatial Fourier transform of the field inside the side-ring. (a) The topological laser exhibits a fixed phase difference between the modes in the right-hand side ring, which gives rise to the mode locking. (b) The trivial laser displays multivalued phase differences between the modes of the same ring, which therefore cannot be synchronized to yield mode-locked operation. The time axis is in units of $\tau=2 \pi / \Omega$.

the topological laser operates through one-way edge state while maintaining high slope efficiency even in the presence of disorder. When the disorder level is strong enough to close the topological band gap, the slope efficiency tends to approach that of a trivial laser. Here, we assume that the disorder is randomly distributed to all the modes in the synthetic space, as described by a tight-binding theory.

The topological edge state propagating in the edges of synthetic space indicates that the light of the laser occupies all the modes in the side resonators, as shown in the yellow shaded regions in Fig. 1(b). The calculated spectra for the topological and trivial lasers under the same level of pumping are shown in Figs. 2(c) and 2(d), respectively. Since the topological laser lases in a single Floquet mode (unlike the trivial laser) that lases in many Floquet modes, the spectrum of the topological laser shows higher and smoother spectrum intensity distributions than that of the trivial laser, which indicates that the light in the topological laser system evolves through all the (pumped) modes in the synthetic dimension. In contrast, for the trivial laser, the light becomes more localized in the frequency dimension, populating only a subgroup of modes. Furthermore, as shown in the right-hand part of Fig. 2(c), the topological laser guarantees single Floquet mode lasing within the topological band gap in the synthetic space, leading to the form of a frequency comb with spacing $\Omega$. On the other hand, in the trivial system lasing is weaker and occurs at multiple Floquet modes, as shown in the right-hand part of Fig. 2(d).

The power flux of the light in the topological cavity is unidirectional. This is shown in Fig. 1(b), where the unidirectionality can be observed by noticing that the power increases while traveling counterclockwise around the topological cavity in synthetic space, and then abruptly drops after coupling to the side resonator. The dispersion relation of the single one-way edge state with the quasienergy within the band gap makes the topological edge state in synthetic space correspond to a single-valued wave vector in frequency dimension. This single-valued wave vector determines the fixed phase difference between the adjacent modes of side resonator [e.g., $0.3 \pi$, green dot in the figure in Sec. A in Supplemental Material [59] corresponding to Fig. 3(a)]. Figure 3(a) shows the result of time-domain simulations of such evolution (with a fixed phase between adjacent modes) for a duration of $800 \tau$ $(\tau=2 \pi / \Omega)$, when the topological laser system arrives at steady state. On the other hand, as shown in Fig. 3(b), in the topologically trivial laser system the phase difference between the modes exhibits multiple values and the power in each is weak, which, unlike the topological case, lacks a single dominant peak. Evidently, in the trivial system the spatial and temporal modes cannot be synchronized to be all in phase periodically in time.

The fixed phase difference between adjacent modes [Fig. 3(a)] hints that the laser operates in a mode-locked fashion. Essentially, the side resonators of the topological module generate a frequency comb with a fixed phase difference between the modes, which give rise to the topologically protected phase locking of the light in all the ring resonators in the system, including the side resonators which lase in multiple modes simultaneously. The lasing modes constructively interfere and produce a periodic train of narrow pulses [Fig 4(a)]. In fact, this mechanism gives rise to active mode locking of the entire laser array system. Figure 4(a) shows the pulses, each of width (at FWHM) of $\sim 0.045 \tau$, separated by $\tau=2 \pi / \Omega$ emitted from the topological lasing system in synthetic dimensions. We find that the topological mode-locked laser generates pulses which are immune to the disorder on the ring resonators, as presented in Fig. 4(b). In contrast to that, the trivial system exhibits multimode lasing and backscattering, which does not produce clean mode-locked pulses even in the absence of disorder [Fig. 4(c)] and becomes worse in the presence of disorder [Fig. 4(d)]. 

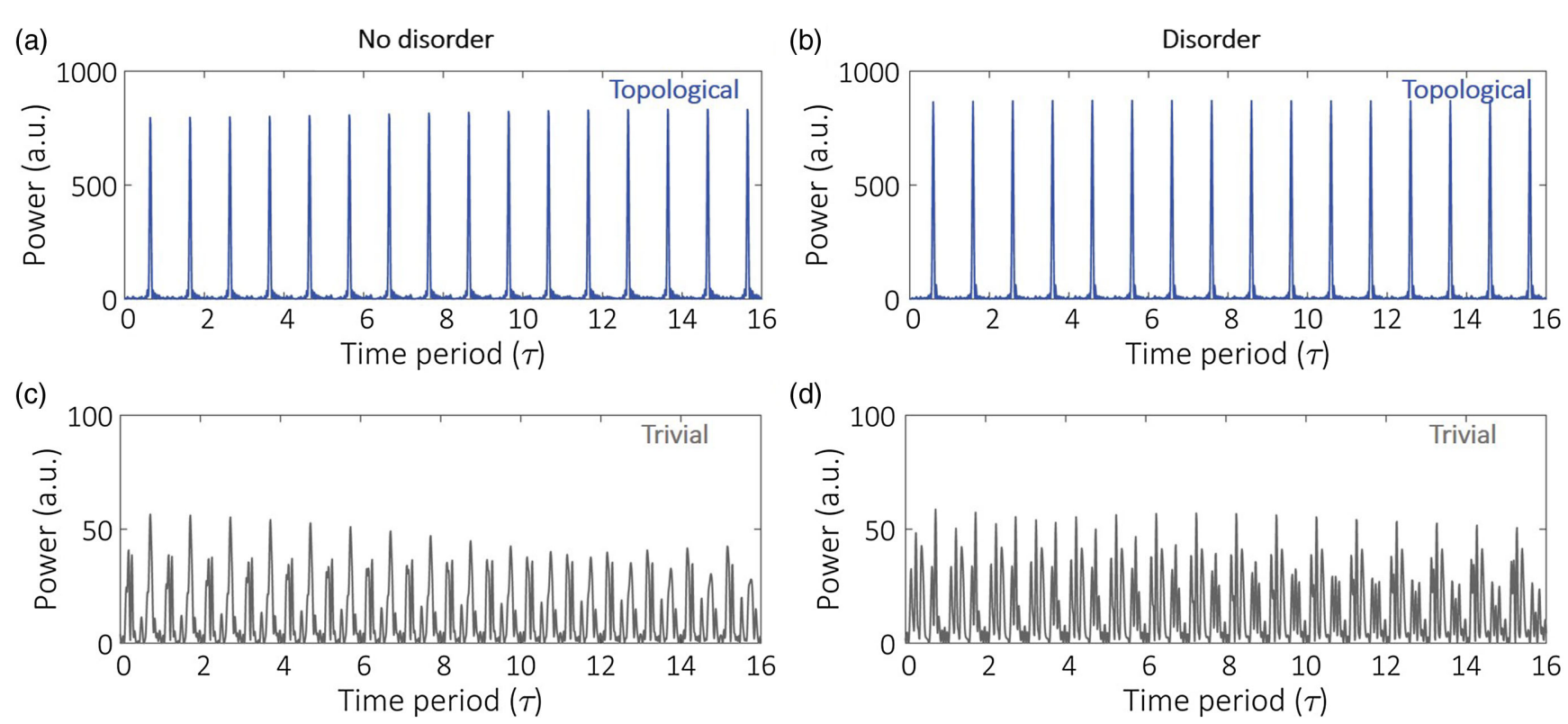

FIG. 4. Temporal dynamics of the laser output power (in arbitrary units, arb. units) emitted from the side resonators. The topological module emits mode-locked pulses (a) and maintains clean mode-locked operation even under considerable disorder with $W=0.5$ (b). On the other hand, the trivial laser system $(\phi=0)$ displays multiple bursts of power, which broaden and fluctuate in the absence $(c)$ and presence $(d)$ of on-site disorder which introduces frequency mismatch among the resonators. The blue and gray curves indicate the results of topological and trivial lasers, respectively. The time axis is in units of $\tau=2 \pi / \Omega$. Note that in the simulations, background noise is present at all times. The background noise is distributed in $[-\delta, \delta]$ and $\delta=0.2$.

Having described a single module of our system, consider now an assembly of modules, as described by Fig. 5(a). Here [Fig. 5(a)], we connect the different modules (each module has 6 rings overall) by disconnecting a single connection of each module and connecting both edges to two neighboring modules. The two remaining edges at the first and last modules are then connected to one another to form a closed loop. In this configuration the width of the bulk of each module is two rings (which we find to be optimal compared to other bulk sizes). The result in synthetic space [Fig. 5(b)] is that there is only one closed path with gain for light. This path is unidirectional due to the topology (the modulation phase) in synthetic space. This is an array of $n$ modules, with two sets of $n$ side rings whose modes are locked at constant phases to yield modelocked pulses from the entire laser diode array. Figure 5(c) presents the intensity of the light emitted from one set of the side resonators as they constructively interfere due to the topological lasing process. Thus, the topological laser array generates a train of clean mode-locked pulses even in the presence of considerable disorder [Fig. 5(e)]. The trivial laser array emits fluctuating pulses with much less output power [Figs. 5(d) and 5(f)] since modes cannot constructively interfere at synchronized times, as they do in a topological laser. The pulse emitting efficiency in the topological device is, for $n=5$ modules, about 50 times higher than that of the trivial laser. These results are further confirmed by verifying that the peak power scales quadratically with the number of emitters, while-had it been incoherent-the peak power would be merely the sum of the time-averaged intensities, which of course would never display clean pulses. Figure 5(g) shows exactly this, and serves as an unequivocal proof that the topological platform can indeed force mode locking of very many semiconductor laser emitters-exactly because it forces a specific (fixed) phase difference between adjacent emitters, whereas for the trivial platform the relative phase between emitters varies fast with time. As such, the light emitted from the topological system interferes coherently at roundtrip times with peak power scaling quadratically with the number of emitters-leading to a train of clean modelocked pulses, while the light from the trivial system cannot do that because the different emitters are not synchronized in their phases.

This naturally raises questions about the reasons: what is it in the topological features that makes the ensemble of semiconductor laser elements mode locked? The answer is twofold. First, the topological edge mode always moves at a constant velocity, which not only forces all the lasers to lock in their set of resonance frequencies, but also gives rise to a constant phase between modes in adjacent resonators. Effectively, the topological landscape makes sure that the system lases in a single Floquet edge mode and the dynamics in the synthetic dimension forces all the resonant frequencies to lock, giving rise to mode-locked pulses. Second, the topological protection of transport endows the system with robustness against disorder, which in a laser array arises from small variations in the resonators, inevitably occurring in the fabrication process. Without the topological features, the assembly lases in multiple 
(a)

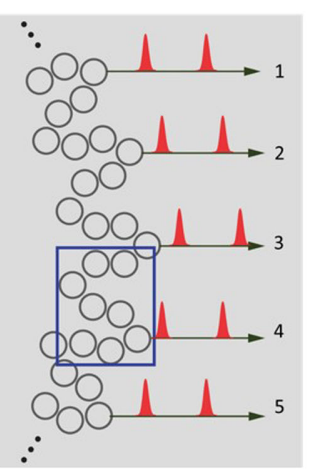

(b)

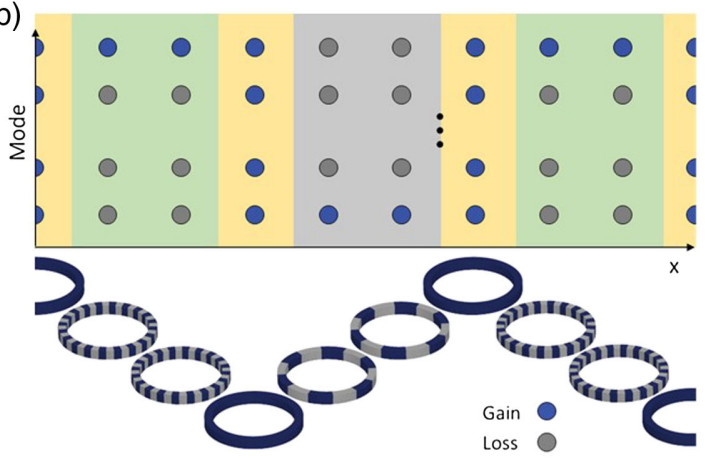

(g)

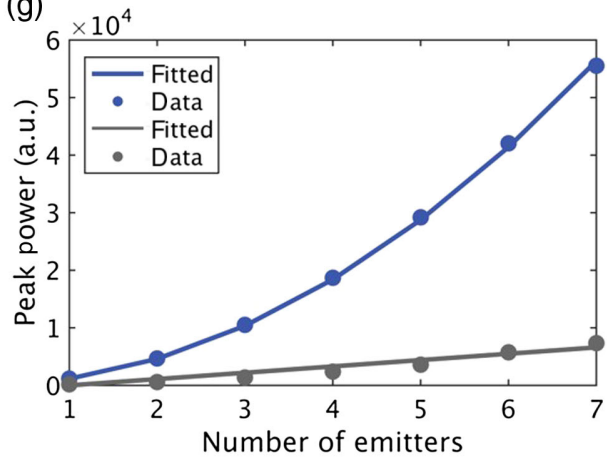

(c)

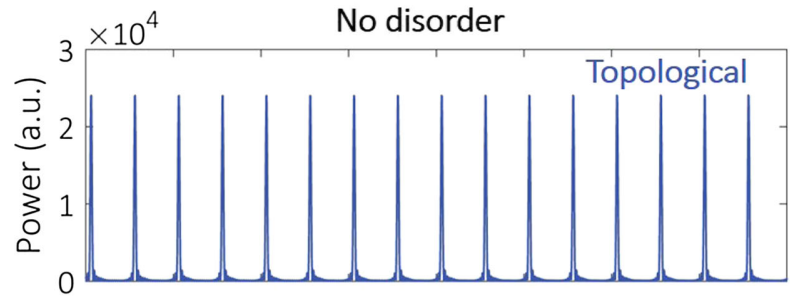

(d)

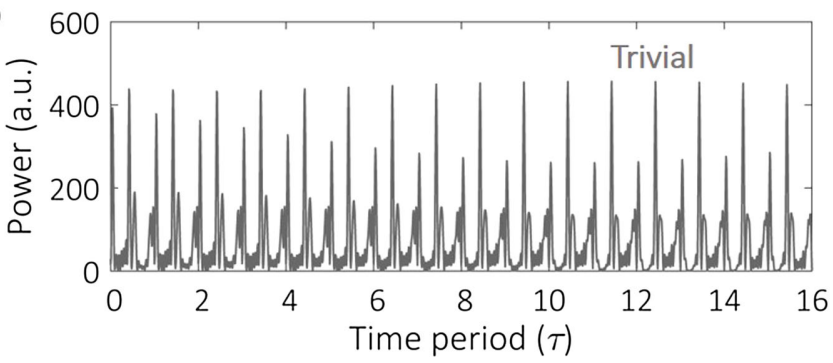

(e)

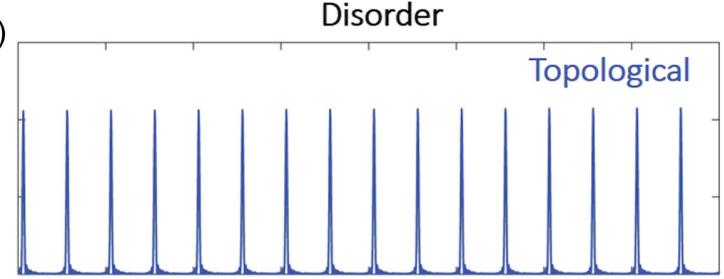

(f)

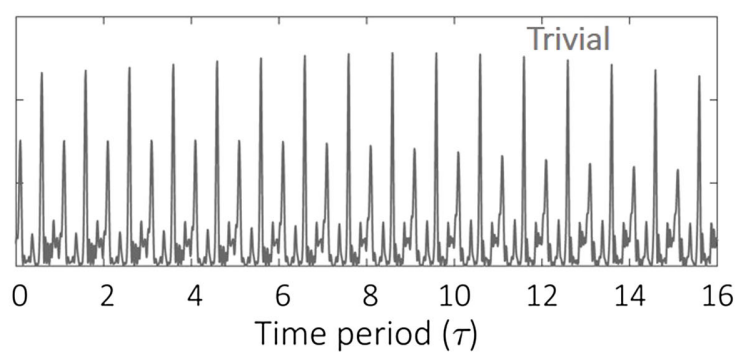

FIG. 5. Topological mode-locked laser array. (a) Scheme showing a laser array of $n=5$ modules [each of which is described by Fig. 1(a)]. The side resonators in the laser array configuration connect different chains of ring resonators. The lower panel shows an enlarged view of the blue box in (a). The laser light is extracted through output couplers. (b) The gain profile in synthetic dimensions. The ring resonators correspond to the ones within the blue box in (a). (c),(e) The topological laser array generates a train of mode-locked pulses (c) which are emitted from the rings with homogenous gain [top or bottom rings in (b)], and remain clean mode-locked pulses even in the presence of considerable on-site disorder with $W=0.3$ (which introduces frequency mismatch among the resonators) (e). (d),(f) The trivial laser array emits fluctuating pulses with much less output power (in arbitrary units, arb. units) even in the absence (d) and presence (f) of disorder. (g) Peak power scales with the number of emitters quadratically for topological laser (blue) and linearly for trivial laser (gray). The simulation parameters we used are the same as those in Fig. 1(a). The time axis is in units of $\tau=2 \pi / \Omega$. In panels (c)-(f) the laser output power is calculated from the sum of the electric fields of side rings in the laser array.

spatial modes which cannot synchronize their resonant frequencies, and hence cannot mode lock.

The system we propose here can be experimentally realized using ring resonators made of InGaAsP quantum wells similar to the rings in Ref. [38]. The synthetic dimension can be constructed in the rings by using electro-optic modulators $[61,62]$ modulating the refractive index. To match the modulation rate to the spacing between adjacent modes of the ring, the ring radius can be about $200 \mu \mathrm{m}$ for a modulation rate of $80 \mathrm{GHz}$, which is accessible with current technology [61]. The relation between the radius and modulation rate is determined by $n_{\text {eff }}(\Omega / c) 2 \pi \mathrm{r}=2 \pi$. This would correspond to $\sim 0.6 \mathrm{ps}$ pulses separated by $\tau=(2 \pi / \Omega)=12.5$ ps. Increasing the number of modes would make the pulses narrower, with the same repetition rate. To facilitate the lasing of the artificial edge and selectively populate the target mode, we can adopt a general design of periodically arranging $\mathrm{Cr}-\mathrm{Ge}$ structures on top of the resonator along the azimuthal direction [63,64]. The specific mode (of a single ring) experiencing maximum gain will reach the lasing threshold first and lase in a single frequency (see more details on the effects of breaking the azimuthal symmetry of the rings in Sec. C in the Supplemental Material [59]). Therefore, we can provide azimuthal gain or loss modulation of a smaller azimuthal order (e.g., 30) to the rings in the lower chain [as in Fig. 1(a)], and modulation with higher azimuthal order (say, 50) to the rings in the upper chain. In this design relying on synthetic-space topology, we combine the upper edge (at mode 50) and the lower edge (at mode 30) in a desirable fashion for active mode locking of a large number of semiconductor laser elements. 
To summarize, we utilized the principles of mode-locked lasers combined with the concept of photonic topological insulators in synthetic dimensions to propose a system that can force a large array of semiconductor laser to emit transform-limited mode-locked pulses. While the design here is suggested as a proof of concept, it can be optimized to a specific laser system. At the heart of the ideas is the unique feature of a topological edge state that forces a fixed phase difference between adjacent sides as it evolves, and the topological protection which provides robustness to disorder and imperfections. Our system here is topological in synthetic space; hence the single Floquet topological edge state in synthetic space forces a fixed phase difference between the multiple modes in side resonators and locks all the elements in the laser array. As a result, the comparison between topological and nontopological structures is dramatic: the topological system emits a train of clean transform-limited pulses whereas the trivial system emits irregular bursts characteristic of modulated multielement lasers that lack locking. Also, as we show in Sec. G in Supplemental Material [59], the small side lobes between the pulse peaks can be further suppressed by including inhomogeneous broadening. Other than emitting modelocked pulses, we also show that a similar topological laser array system, where the laser utilizes the gain from all the ring resonators, can be designed to have single-frequency lasing in synthetic dimensions (see details in Sec. G in Supplemental Material [59]). We envision many more possibilities relying on utilizing topological phenomena in synthetic dimensions in lasers and amplifiers, suggesting a new route for developing active photonic devices.

\section{ACKNOWLEDGMENTS}

This work was sponsored by the Israel Science Foundation and by an Advanced Grant from the European Research Council. The authors acknowledge excellent discussions with Professor Gadi Eisenstein of the Technion on modulated semiconductor lasers and active mode locking.

[1] M. Z. Hasan and C. L. Kane, Colloquium: Topological Insulators, Rev. Mod. Phys. 82, 3045 (2010).

[2] X.-L. Qi and S.-C. Zhang, Topological Insulators and Superconductors, Rev. Mod. Phys. 83, 1057 (2011).

[3] M. Konig, S. Wiedmann, C. Brune, A. Roth, H. Buhmann, L. W. Molenkamp, X.-L. Qi, and S.-C. Zhang, Quantum Spin Hall Insulator State in HgTe Quantum Wells, Science 318, 766 (2007).

[4] F. D. M. Haldane and S. Raghu, Possible Realization of Directional Optical Waveguides in Photonic Crystals with Broken Time-Reversal Symmetry, Phys. Rev. Lett. 100, 013904 (2008).

[5] Z. Wang, Y.D. Chong, J. D. Joannopoulos, and M. Soljačić, Reflection-Free One-Way Edge Modes in a
Gyromagnetic Photonic Crystal, Phys. Rev. Lett. 100, 013905 (2008).

[6] Z. Wang, Y. Chong, J. D. Joannopoulos, and M. Soljačić, Observation of Unidirectional Backscattering-Immune Topological Electromagnetic States, Nature (London) 461, 772 (2009).

[7] T. Ozawa, H. M. Price, A. Amo, N. Goldman, M. Hafezi, L. Lu, M. C. Rechtsman, D. Schuster, J. Simon, O. Zilberberg, and I. Carusotto, Topological Photonics, Rev. Mod. Phys. 91, 015006 (2019).

[8] M. Hafezi, E. A. Demler, M. D. Lukin, and J. M. Taylor, Robust Optical Delay Lines with Topological Protection, Nat. Phys. 7, 907 (2011).

[9] K. Fang, Z. Yu, and S. Fan, Realizing Effective Magnetic Field for Photons by Controlling the Phase of Dynamic Modulation, Nat. Photonics 6, 782 (2012).

[10] A. B. Khanikaev, S. H. Mousavi, W.-K. Tse, M. Kargarian, A. H. MacDonald, and G. Shvets, Photonic Topological Insulators, Nat. Mater. 12, 233 (2013).

[11] M. C. Rechtsman, J. M. Zeuner, Y. Plotnik, Y. Lumer, D. Podolsky, F. Dreisow, S. Nolte, M. Segev, and A. Szameit, Photonic Floquet Topological Insulators, Nature (London) 496, 196 (2013).

[12] M. Hafezi, S. Mittal, J. Fan, A. Migdall, and J. M. Taylor, Imaging Topological Edge States in Silicon Photonics, Nat. Photonics 7, 1001 (2013).

[13] G. Q. Liang and Y. D. Chong, Optical Resonator Analog of a Two-Dimensional Topological Insulator, Phys. Rev. Lett. 110, 203904 (2013).

[14] L.-H. Wu and X. Hu, Scheme for Achieving a Topological Photonic Crystal by Using Dielectric Material, Phys. Rev. Lett. 114, 223901 (2015).

[15] C. He, X. Ni, H. Ge, X.-C. Sun, Y.-B. Chen, M.-H. Lu, X.-P. Liu, and Y.-F. Chen, Acoustic Topological Insulator and Robust One-Way Sound Transport, Nat. Phys. 12, 1124 (2016).

[16] X. Zhang, M. Xiao, Y. Cheng, M.-H. Lu, and J. Christensen, Topological Sound, Commun. Phys. 1, 97 (2018).

[17] R. Süsstrunk and S. D. Huber, Observation of Phononic Helical Edge States in a Mechanical Topological Insulator, Science 349, 47 (2015).

[18] S. D. Huber, Topological Mechanics, Nat. Phys. 12, 621 (2016).

[19] M. C. Rechtsman, J. M. Zeuner, A. Tünnermann, S. Nolte, M. Segev, and A. Szameit, Strain-Induced Pseudomagnetic Field and Photonic Landau Levels in Dielectric Structures, Nat. Photonics 7, 153 (2013).

[20] J. Koch, A. A. Houck, K. Le Hur, and S. M. Girvin, TimeReversal-Symmetry Breaking in Circuit-QED-Based Photon Lattices, Phys. Rev. A 82, 043811 (2010).

[21] R. O. Umucalılar and I. Carusotto, Artificial Gauge Field for Photons in Coupled Cavity Arrays, Phys. Rev. A 84, 043804 (2011).

[22] O. Boada, A. Celi, J. I. Latorre, and M. Lewenstein, Quantum Simulation of an Extra Dimension, Phys. Rev. Lett. 108, 133001 (2012).

[23] A. Celi, P. Massignan, J. Ruseckas, N. Goldman, I. B. Spielman, G. Juzeliūnas, and M. Lewenstein, Synthetic Gauge Fields in Synthetic Dimensions, Phys. Rev. Lett. 112, 043001 (2014). 
[24] T. Ozawa and H. M. Price, Topological Quantum Matter in Synthetic Dimensions, Nat. Rev. Phys. 1, 349 (2019).

[25] M. Mancini, G. Pagano, G. Cappellini, L. Livi, M. Rider, J. Catani, C. Sias, P. Zoller, M. Inguscio, M. Dalmonte, and L. Fallani, Observation of Chiral Edge States with Neutral Fermions in Synthetic Hall Ribbons, Science 349, 1510 (2015).

[26] B. K. Stuhl, H. I. Lu, L. M. Aycock, D. Genkina, and I. B. Spielman, Visualizing Edge States with an Atomic Bose Gas in the Quantum Hall Regime, Science 349, 1514 (2015).

[27] H. M. Price, T. Ozawa, and N. Goldman, Synthetic Dimensions for Cold Atoms from Shaking a Harmonic Trap, Phys. Rev. A 95, 023607 (2017).

[28] D. Jukić and H. Buljan, Four-Dimensional Photonic Lattices and Discrete Tesseract Solitons, Phys. Rev. A 87, 013814 (2013).

[29] Y. E. Kraus, Y. Lahini, Z. Ringel, M. Verbin, and O. Zilberberg, Topological States and Adiabatic Pumping in Quasicrystals, Phys. Rev. Lett. 109, 106402 (2012).

[30] O. Zilberberg, S. Huang, J. Guglielmon, M. Wang, K. P. Chen, Y. E. Kraus, and M. C. Rechtsman, Photonic Topological Boundary Pumping as a Probe of 4D Quantum Hall Physics, Nature (London) 553, 59 (2018).

[31] X.-W. Luo, J.-S. Xu, G.-C. Guo, X. Zhou, C.-F. Li, and Z.-W. Zhou, Quantum Simulation of 2D Topological Physics in a 1D Array of Optical Cavities, Nat. Commun. 6, 7704 (2015).

[32] T. Ozawa, H. M. Price, N. Goldman, O. Zilberberg, and I. Carusotto, Synthetic Dimensions in Integrated Photonics: From Optical Isolation to Four-Dimensional Quantum Hall Physics, Phys. Rev. A 93, 043827 (2016).

[33] L. Yuan, Y. Shi, and S. Fan, Photonic Gauge Potential in a System with a Synthetic Frequency Dimension, Opt. Lett. 41, 741 (2016).

[34] Q. Lin, M. Xiao, L. Yuan, and S. Fan, Photonic Weyl Point in a Two-Dimensional Resonator Lattice with a Synthetic Frequency Dimension, Nat. Commun. 7, 13731 (2016).

[35] E. Lustig, S. Weimann, Y. Plotnik, Y. Lumer, M. A. Bandres, A. Szameit, and M. Segev, Photonic Topological Insulator in Synthetic Dimensions, Nature (London) 567, 356 (2019).

[36] A. Dutt, Q. Lin, L. Yuan, M. Minkov, M. Xiao, and S. Fan, A Single Photonic Cavity with Two Independent Physical Synthetic Dimensions, Science 367, 59 (2020).

[37] G. Harari, M. A. Bandres, Y. Lumer, M. C. Rechtsman, Y. D. Chong, M. Khajavikhan, D. N. Christodoulides, and M. Segev, Topological Insulator Laser: Theory, Science 359, eaar4003 (2018).

[38] M. A. Bandres, S. Wittek, G. Harari, M. Parto, J. Ren, M. Segev, D. N. Christodoulides, and M. Khajavikhan, Topological Insulator Laser: Experiments, Science 359, eaar4005 (2018).

[39] B. Bahari, A. Ndao, F. Vallini, A. El Amili, Y. Fainman, and B. Kanté, Nonreciprocal Lasing in Topological Cavities of Arbitrary Geometries, Science 358, 636 (2017).

[40] Z.-K. Shao, H.-Z. Chen, S. Wang, X.-R. Mao, Z.-Q. Yang, S.-L. Wang, X.-X. Wang, X. Hu, and R.-M. Ma, A High-Performance Topological Bulk Laser Based on
Band-Inversion-Induced Reflection, Nat. Nanotechnol. https://doi.org/10.1038/s41565-019-0584-x (2019).

[41] Y. Zeng et al., Electrically Pumped Topological Laser with Valley Edge Modes, Nature (London) 578, 246 (2020).

[42] J. P. Gordon, H. J. Zeiger, and C. H. Townes, The MaserNew Type of Microwave Amplifier, Frequency Standard, and Spectrometer, Phys. Rev. 99, 1264 (1955).

[43] P. A. Fokker, J. I. Dijkhuis, and H. W. de Wijn, Stimulated Emission of Phonons in an Acoustical Cavity, Phys. Rev. B 55, 2925 (1997).

[44] D. J. Bergman and M. I. Stockman, Surface Plasmon Amplification by Stimulated Emission of Radiation: Quantum Generation of Coherent Surface Plasmons in Nanosystems, Phys. Rev. Lett. 90, 027402 (2003).

[45] M. A. Noginov, G. Zhu, A. M. Belgrave, R. Bakker, V. M. Shalaev, E. E. Narimanov, S. Stout, E. Herz, T. Suteewong, and U. Wiesner, Demonstration of a Spaser-Based Nanolaser, Nature (London) 460, 1110 (2009).

[46] R. F. Oulton, V. J. Sorger, T. Zentgraf, R.-M. Ma, C. Gladden, L. Dai, G. Bartal, and X. Zhang, Plasmon Lasers at Deep Subwavelength Scale, Nature (London) 461, 629 (2009).

[47] S. Klembt, T. H. Harder, O. A. Egorov, K. Winkler, R. Ge, M. A. Bandres, M. Emmerling, L. Worschech, T. C. H. Liew, M. Segev, C. Schneider, and S. Höfling, ExcitonPolariton Topological Insulator, Nature (London) 562, 552 (2018).

[48] H. Suchomel, S. Klembt, T. H. Harder, M. Klaas, O. A. Egorov, K. Winkler, M. Emmerling, R. Thomale, S. Höfling, and C. Schneider, Platform for Electrically Pumped Polariton Simulators and Topological Lasers, Phys. Rev. Lett. 121, 257402 (2018).

[49] E. Kapon, J. Katz, and A. Yariv, Supermode Analysis of Phase-Locked Arrays of Semiconductor Lasers, Opt. Lett. 9 , 125 (1984).

[50] J. K. Butler, D. E. Ackley, and D. Botez, Coupled-Mode Analysis of Phase-Locked Injection Laser Arrays, Appl. Phys. Lett. 44, 293 (1984).

[51] P. Vasil'ev, Ultrafast Diode Lasers: Fundamentals and Applications (Artech House Inc., Norwood, 1995).

[52] C. H. Henry, Theory of the Linewidth of Semiconductor Lasers, IEEE J. Quantum Electron. 18, 259 (1982).

[53] C. Harder, K. Vahala, and A. Yariv, Measurement of the Linewidth Enhancement Factor $\alpha$ of Semiconductor Lasers, Appl. Phys. Lett. 42, 328 (1983).

[54] J. P. van der Ziel, H. Temkin, R. D. Dupuis, and R. M. Mikulyak, Mode-Locked Picosecond Pulse Generation from High Power Phase-Locked GaAs Laser Arrays, Appl. Phys. Lett. 44, 357 (1984).

[55] M. Segev, Y. Ophir, B. Fischer, and G. Eisenstein, Mode Locking and Frequency Tuning of a Laser Diode Array in an Extended Cavity with a Photorefractive Phase Conjugate Mirror, Appl. Phys. Lett. 57, 2523 (1990).

[56] A. Mar, R. Helkey, T. Reynolds, J. Bowers, D. Botez, C. Zmudzinski, C. Tu, and L. Mawst, Mode-Locked Multisegment Resonant-Optical-Waveguide Diode Laser Arrays, IEEE Photonics Technol. Lett. 5, 1355 (1993).

[57] L. Yuan, Q. Lin, M. Xiao, and S. Fan, Synthetic Dimension in Photonics, Optica 5, 1396 (2018). 
[58] D. R. Hofstadter, Energy Levels and Wave Functions of Bloch Electrons in Rational and Irrational Magnetic Fields, Phys. Rev. B 14, 2239 (1976).

[59] See Supplemental Material at http://link.aps.org/ supplemental/10.1103/PhysRevX.10.011059 for additional numerical calculations on the topological nature and the robustness of the mode-locked topological insulator laser in synthetic dimensions.

[60] L. Yuan, Q. Lin, A. Zhang, M. Xiao, X. Chen, and S. Fan, Photonic Gauge Potential in One Cavity with Synthetic Frequency and Orbital Angular Momentum Dimensions, Phys. Rev. Lett. 122, 083903 (2019).

[61] L. D. Tzuang, M. Soltani, Y. H. D. Lee, and M. Lipson, High RF Carrier Frequency Modulation in Silicon
Resonators by Coupling Adjacent Free-Spectral-Range Modes, Opt. Lett. 39, 1799 (2014).

[62] A. Dutt, M. Minkov, Q. Lin, L. Yuan, D. A. B. Miller, and S. Fan, Experimental Band Structure Spectroscopy along a Synthetic Dimension, Nat. Commun. 10, 3122 (2019).

[63] L. Feng, Z. J. Wong, R.-M. R. M. Ma, Y. Wang, and X. Zhang, Single-Mode Laser by Parity-Time Symmetry Breaking, Science 346, 972 (2014).

[64] P. Miao, Z. Zhang, J. Sun, W. Walasik, S. Longhi, N. M. Litchinitser, and L. Feng, Orbital Angular Momentum Microlaser, Science 353, 464 (2016). 